\title{
Frustration of total internal reflection by a hidden nanowire
}

\author{
Leonid Frumin ${ }^{1,2}$, Anton Nemykin ${ }^{1,2}$, and David Shapiro ${ }^{1,2, *}$ \\ ${ }^{1}$ Institute of Automation and Electrometry, SB RAS, 630090 Novosibirsk, Russia \\ ${ }^{2}$ Novosibirsk State University, 630090 Novosibirsk, Russia
}

\begin{abstract}
A metallic wire embedded in the dielectric substrate leads to frustration of the total internal reflection at the interface between dielectric and free space. The scattering by a gold cylinder is studied in order to exploit the light for hidden objects optical diagnostics. The p-wave incidenting by the angle close to total reflection with the frequency near plasmonic resonance is treated by the modified boundary elements method. The magnetic and electric fields at the interface are calculated. The scattering diagrams are found in near and far field.
\end{abstract}

The scattering of evanescent wave by particles is important elementary process of nearfield optics $[1,2]$. It is a particularly promising method in near-field scanning microscopy [3-5]. The interesting case of metallic particle embedded in dielectric half-space has a number of specific technical applications, for instance, the tomographic reconstruction of hidden scatterer, see [6] and references therein. As distinct from a running wave, the evanescent wave is not a limited solution of Maxwell equations in the whole space. That is why its scattering should be considered along with its source, in our problem the dielectric half-space with incident and reflecting waves. For calculation we exploit the modified boundary elements method (BEM) [7]. The feature of modified BEM is the special Green function exactly taking into account the layered medium (free and dielectric half-spaces). The substitution of Green function makes it possible to use the same numerical scheme as for a running wave. The discretization of infinite boundary is not necessary. Multiple scattering and reflections are accounted automatically. Moreover, the modified BEM has a number of advantages compared to other common methods based on the finite differences or finite elements. BEM reduces the problem to calculation of field at the boundary, then decrements, in fact, the dimension of problem. In our case two-dimension problem reduces to one-dimension boundary integral equations.

In previous work $[7,8]$ the object is located on the substrate. In present paper the scatterer hidden inside the dielectric is considered. The object is illuminated by the incident wave near the angle close to total internal reflection (TIR). In this case TIR is frustrated by the scattering and strongly depends on the scatterer parameters. The goal is to study the angular diagram of scattering.

The $p$-wave is considered where the magnetic field has one component along the axis of cylinder. The calculations are performed for gold cylinder with radius $150,100,75,50 \mathrm{~nm}$

* Corresponding author: shapiro@iae.nsk.su 
at distance 50, 100 and $150 \mathrm{~nm}$ from the interface between glass (dielectric permittivity 2.25) and free space. The incidence angle $\theta$ is close to TIR angle $41.8^{0}$ at the chosen wavelength $\lambda=1.512 \mu \mathrm{m}$. Fig. 1 illustrates results for cylinder of radius $150 \mathrm{~nm}$ at distance $50 \mathrm{~nm}$.

Fig. 1(a) shows the angular diagram of the energy flux of scattered wave, averaged over a period, at the circles with different radius $R$ at $\theta=41^{\circ}$. The major part of radiation is reflected back into the glass with angles $180-360^{\circ}$. Only a small part of light is scattered into free space $0-180^{\circ}$. In free space the main part enters the first quadrant $0-90^{\circ}$, Fig. 1(b). Above the TIR angle indicatrix swings to the second quadrant $90-180^{\circ}$ and the "backward scattering" prevails, Fig. 1(c), due to the scattering of wave reflected from the plane.
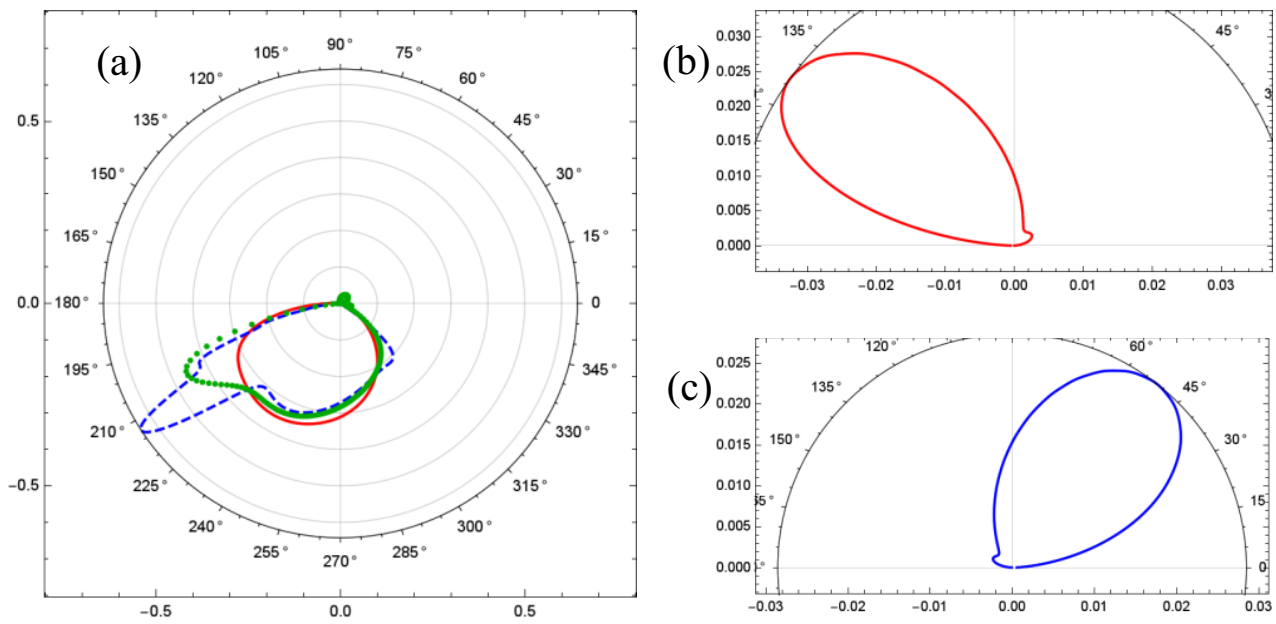

Fig. 1. (a) Scattering indicatrix at $\mathrm{R} / \lambda=1$ (solid curve), 2.5 (dots), 10 (dashes). The magnified angular diagram in the free space at $\mathrm{R} / \lambda=10$ (far field) and $\theta=41^{\circ}(\mathrm{b}), 43^{\circ}(\mathrm{c}$ ).

The signal of frustration in the upper half-plane, where a detector is supposed, turns to be sufficiently weak. There is a way to increase the scattering using a periodic grid instead of single wire. This case can be analyzed by the effective Green function for periodic structure [8].

The Russian Foundation for Basic Research (16-02-00511) and Government program of the leading research schools (NSh-6898.2016.2) support this work.

\section{References}

1. L. Novotny, B. Hecht, Principles of Nano-Optics (Cambridge, 2006)

2. M.I. Stockman, Phys. Today 64, 39 (2011)

3. B. Hecht, B. Sick et al., J. Chem. Phys. 112, 7761 (2000)

4. C. Girard, C. Joachim, S. Gauthier, Rep. Prog. Phys. 63, 893 (2000)

5. P.S. Carney, J.C. Schotland, Opt. Lett. 26, 1072 (2001)

6. T. Yamaoki, H. Hamada, O. Matoba, Appl. Opt. 55, 6874 (2016)

7. O.V. Belai, L.L. Frumin et al., Opt. Lett. 36, 954 (2011)

8. L.L. Frumin, A.V. Nemykin et al., J. Opt. 15, 085002 (2013) 